\title{
An Autonomous Resource Block Assignment Scheme for OFDMA-Based Relay-Assisted Cellular Networks
}

\author{
Yaser M. M. Fouad, Ramy H. Gohary, and Halim Yanikomeroglu
}

\begin{abstract}
Terminal relaying offers an effective means for improving the performance of OFDMA-based wireless networks. However, with the increase in the number of relaying terminals (RTs), their coordination becomes a cumbersome task. To address this drawback, in this paper an autonomous scheme is proposed whereby the RTs assign resource blocks (RBs) to incoming wireless terminals (WTs) in a way that minimizes the number of hit occurrences at which the same $R B$ is assigned to multiple WTs. The proposed scheme uses cyclic group generators to determine the sequence of RBs to be assigned by each RT. This scheme is particularly beneficial in terminal relaying systems in which the distribution of the WTs is nonuniform and the channel quality indicators are not available. Simulation results show that the proposed scheme performs significantly better than currently available autonomous assignment schemes.
\end{abstract}

Index Terms-Terminal relaying, medium access control schemes, distributed resource block coordination, cyclic groups.

\section{INTRODUCTION}

T HE utilization of relays in wireless data networks enhances coverage [1] and has a significant impact on the maximum data throughput that these networks can communicate. For systems in which the relays are fixed and their number is small, it is plausible to assume that the locations of the relays are chosen to provide good average performance [2] and that the overhead required for their centralized coordination [3] at the base station (BS) is negligible.

Although fixed relays enhance the performance of cellular networks, their efficacy becomes less pronounced with the increase in the number of wireless terminals (WTs) with high data rate requirements. This is because with the increase in the number of WTs, maintaining a certain quality of service requires increasing the number of fixed relays, which subsequently increases the cost of the network and the coordination overhead. These difficulties can be mitigated by employing terminal relaying wherein idle terminals cooperate with active ones to improve the performance of the network. Since, at a given time instant, a considerable portion of WTs is likely to be idle, the prospective increase in the overall number of WTs will result in a large number of relaying

Manuscript received October 18, 2010; revised March 1, 2011 and August 31, 2011; accepted October 21, 2011. The associate editor coordinating the review of this paper and approving it for publication was Y.-C. Ko.

The authors are with the Department of Systems and Computer Engineering, Carleton University, Ottawa, Ontario, Canada (e-mail: \{yfouad, gohary, halim\}@sce.carleton.ca).

This work is supported by Research In Motion (RIM) and The Natural Sciences and Engineering Research Council of Canada (NSERC).

Digital Object Identifier 10.1109/TWC.2011.122211.101840 terminals (RTs). However, the large number of these terminals and the incidental manner in which they are likely to operate render their coordination significantly more cumbersome than the coordination of their fixed counterparts. Hence, it can be seen that although terminal relaying overcomes some of the limitations of fixed relaying, it requires significantly more efficient coordination mechanisms.

A problem that arises in relay-assisted OFDMA-based communication systems is how the resource blocks (RBs) are assigned to the set of WTs that are assisted by a particular relay. When the relays are fixed and the channel quality indicators (CQIs) are available, the BSs can assign the RBs in a way that optimizes the network performance. However, this task becomes inefficient in terminal relaying scenarios with a large number of RTs. A more practical approach is to assign the RBs in a distributed manner whereby the RTs autonomously select the RBs of the WTs that they assist.

Most of the existing resource assignment techniques are extensible to relay-assisted communication scenarios. For instance, for OFDMA-based systems with fixed relays, the network throughput can be improved by using a static resource assignment technique known as Fractional Frequency Reuse [4]. Being static, this technique is not suitable for terminal relaying schemes in which the number and location of RTs and the distribution of the wireless terminals vary with time. A more flexible resource assignment scheme is proposed in [3]. When this scheme is applied in relay-assisted networks, each RT chooses its initial assignments randomly and independently of other RTs. Based on the CQIs of the wireless channel, the assignments are updated, either in a centralized or a distributed manner. In the absence of centralized coordination and CQIs, this strategy may result in undesirable instances at which one RB is used by many WTs while other RBs are not used. Such instances result in high, yet avoidable, intracell interference. In contrast with the OFDMA-based systems in [3] and [4], Frequency Hopping (FH) systems in which the WTs hop across frequency slots are considered in [5][8]. In these systems, the frequency slot occupied by each WT at a given dwell interval is determined by a prescribed pseudonoise (PN) based hopping pattern. When the CQIs of the WTs are available, the hopping patterns can be updated to improve the network throughput; see e.g., [5]-[8].

In this paper we consider the problem of assigning RBs in relay-assisted OFDMA-based cellular systems that do not employ hopping techniques. In particular, we propose a scheme for minimizing intra-cell interference by reducing the number 
of hit occurrences; that is, occurrences at which an RB is assigned to multiple WTs.

The scheme proposed herein is autonomous and hence, particularly suited for terminal relaying systems. This is because, in these systems, rapid channel variations may cause the WTs to frequently update their serving RTs, resulting in rapid variations in the network topology. These variations render timely acquisition of the CQI information difficult and impede the implementation of centralized CQI-dependent assignment schemes. Being autonomous, the proposed scheme enables more efficient exploitation of the available spectrum than centralized schemes.

In the proposed scheme, each RT is entitled to use as many RBs as needed; a feature that enables the RTs to efficiently adapt to bursty traffic and nonuniform WT distributions without coordination. To achieve this goal, the set of available RBs is endowed with a cyclic group structure whereby a single group generator spans all possible RBs. Each RT generates its distinct assignment sequence using a prescribed groupgenerator-cyclic-shift pair. This feature relieves the computational burden and the communication overhead required for finding and updating the RB assignments when new WTs access the system. We will show that by careful selection of the group generators and the cyclic shifts, we will be able to obtain assignment sequences that yield a significant reduction in the number of hit occurrences when compared with the random assignment scheme in [3] and the assignment schemes based on the PN sequences used in FH systems. We will show that, when hit occurrences are detectable, using our sequences, the hit sources can be identified and future hits can be avoided, yielding further reduction in the number of hits.

This paper is organized as follows: In Section II relevant facts about cyclic groups are reviewed. The system model is presented in Section III. The proposed scheme for designing the sequences is provided in Section IV, and a technique for identifying the hit source and avoiding future hits is provided in Section V. In Section VI numerical examples are provided and Section VII concludes the paper.

\section{Preliminaries of CyClic Groups}

A group is a set $G$ on which a group operation (denoted by juxtaposition) is defined such that the following closure property is satisfied: For all $(x, y) \in G \times G$ the element $x y \in G$. Additionally, for every element $x \in G$, the inverse $x^{-1} \in G$. This together with the closure property ensure that the identity element $e \in G$; see e.g., [9]. For example, the set $\{0, \ldots, n-1\}$ forms a group under modulo- $n$ addition.

A group $G$ is said to be cyclic if all the elements of the group can be generated by one of its elements. Such an element is known as a group generator. For a group of order $n$, the number of group generators is given by the Euler Totient function, $\phi(n)$, which is defined as the cardinality of the set of integers that are coprime with $n$; that is, $\phi(n)$ is the cardinality of the set $\{q<n \mid \operatorname{gcd}(q, n)=1\}$, where $\operatorname{gcd}(q, n)$ is the greatest common divisor of $q$ and $n$ [9].

A classic result in group theory asserts that all cyclic groups of order $n$ are isomorphic, irrespective of the group operation [10]. Hence, without loss of generality, we will focus on multiplicative cyclic groups formed by the set of integers $\{1, \ldots, n-1\}$.

\section{Cyclic Multiplicative Groups}

A cyclic multiplicative group is a cyclic group in which the group operation is modulo- $n$ multiplication. That is, for every $x, y \in G, x y(\bmod n) \in G$. In fact, the set $\{1, \ldots, n-1\}$ forms a cyclic multiplicative group under modulo- $n$ multiplication if and only if $n$ is a prime number [9].

Let $P$ be a prime number. A primitive root of $P$ is defined to be an integer $g \in\{1, \ldots, P-1\}$ such that the ordered set $\left(g^{k_{1}}(\bmod P), \ldots, g^{k_{p-1}}(\bmod P)\right)=(1, \ldots, P-1)$, for some integers $k_{i} \in\{1, \ldots P-1\}, i=1, \ldots, P-1$. It can be shown that primitive roots are the group generators of multiplicative cyclic groups and their number is given by $\phi(P-1)$.

Primitive roots can be found by efficient techniques and are tabulated for the first few thousand prime numbers. As an illustrative example, when $P=7$ there are $\phi(6)=2$ primitive roots which are $g_{1}=3$ and $g_{2}=5$. Direct evaluation of $g_{i}^{k_{1}}(\bmod P), k_{1}=1, \ldots, 6, i=1,2$ yields the sequences $(3,2,6,4,5,1)$ and $(5,4,6,2,3,1)$, respectively. For relatively small primes, primitive roots can be obtained by searching for $g \in\{1, \ldots, P-1\}$ such that the sequence $\left\{g^{k}(\bmod P)\right\}_{k=1}^{P-1}$ spans all integers from 1 to $P-1$.

Finally, we present a result that will be used to expand the set of sequences that can be generated by primitive roots

Lemma 1: Let $G$ be a cyclic multiplicative group and let $g_{1}$ and $g_{2}$ be two distinct generators modulo- $P$. Then, there is no cyclic shift for which the sequence generated by $g_{1}$ coincides with the sequence generated by $g_{2}$.

Proof: See the Appendix A.

This result implies that cyclic shifts enrich the design of sequences by an additional degree of freedom that cannot be obtained from primitive roots alone.

\section{System Model And Problem Definition}

We consider the relay-assisted OFDMA-based cellular network depicted in Figure 1. In this figure, a set of WTs assume the role of RTs to assist the communication between the BS and other WTs. To act as RTs, the WTs switch to the relaying mode as soon as they become idle, which eliminates the need for the BSs to centrally designate the RTs. Each BS has $N$ RBs at its disposal, is assisted by $M$ RTs, and serves $K$ active WTs. The WTs select their serving RTs based on the strength of the signals of their control channels, irrespective of the coverage area or the cell size.

Each RT has access to all the $N$ RBs of the BS. The assignment of RBs for the BS-RT links is assumed to be performed by the BS in a centralized manner, which is a reasonable assumption given the typically high processing capabilities of the BS. Hence, our focus herein is on the autonomous assignment of RBs for communication over the RT-WT and WT-RT links. By restricting our attention to the case in which $N=P-1$, where $P$ is a prime integer, we will be able to invoke the cyclic group structure described in Section II. Note that, in general, this assumption is not overly restrictive because primes are denser than squares in the set of integers [11]. For the case in which the number of RBs in the system $N$ is not equal to a prime minus one, the closest prime $P \geq N+1$ can be used and the entries larger than $N$ can be skipped in the assignment. In this case, it is 


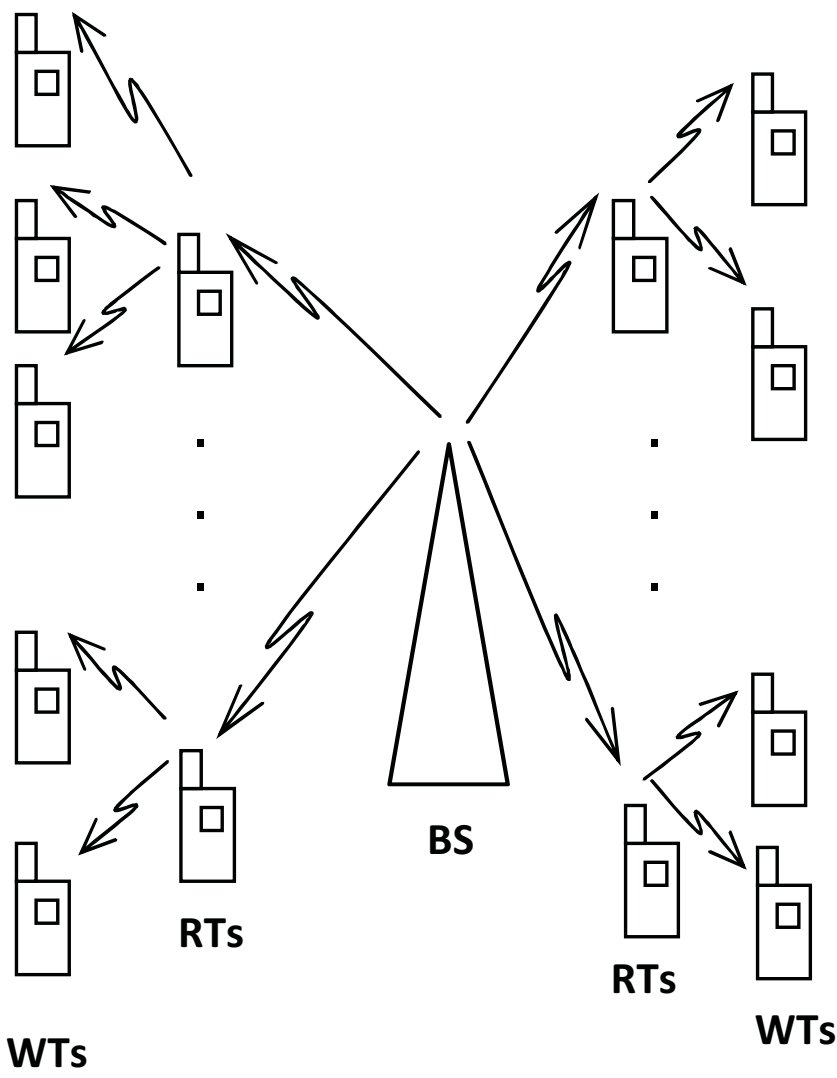

Fig. 1. System model: Idle terminals act as relays to assist active WTs.

also possible to employ other group structures; e.g., the group structure used for generating PN sequences. However, those group structures are typically more complicated to generate and optimize. For instance, PN-based assignments require the search over primitive polynomials. In contrast, for cyclic multiplicative groups, it is only required to take modulo- $P$ of the powers of the primitive roots of $P$.

The WTs access the system one at a time according to a counting process [12]; i.e., the number of WTs in the entire system increases at succedent discrete time instants. This assumption is necessary to ensure that the first RT to assign a particular RB retains it when an assignment conflict occurs. In this situation, the conflicting RTs will refrain from using this RB. Each RT can be loaded by an arbitrary number of WTs independently of the load of the other RTs. It will be observed in the RB assignment scheme described hereinafter that the WTs to be served by the same RT can access the system simultaneously. Using this observation, it is possible to assign multiple RBs to WTs with high traffic requirements.

An RT is selected by the incoming WT depending on its observed signal strength. However, as the wireless channel between the RT and the WT undergoes rapid variations, this choice is frequently updated resulting in a highly dynamic network topology. Furthermore, the backhaul link between the RTs and the BS also contribute to the variations in the network topology. For example, if an RT experiences deep fading on the backhaul link, both the BS and the RT will be able to detect this occurrence and the RT will subsequently notify the WTs to update their RT selection. The RTs assign the RBs to the incoming WTs without knowing their respective CQIs. There is no coordination among the RTs and the assignment of RBs is not centrally controlled by the BS. This necessitates that each RT makes its assignment decisions autonomously. In such a scenario, the RTs will not be able to identify the RBs that can be reused without incurring high intra-cell interference. Hence, the number of WTs, $K$, must not exceed the number of available RBs, $N$; i.e., $K \leq N$.

When a particular RB is assigned to multiple WTs, the intra-cell interference levels observed by these WTs increase, resulting in a significant deterioration of their signal quality. Such an occurrence is undesirable and will be referred to as a 'hit'. Assuming that the number of WTs does not exceed the number of available RBs, the objective of each RT is to assign the RBs in a way that minimizes the number of hits.

For illustration, we provide a two-RT example for an RB assignment scheme that yields a minimum number of hits. Assume that each RT has access to the same set of $N$ RBs. We will denote this set by $\mathcal{R}=\left\{r_{1}, \ldots, r_{N}\right\}$. The first RT assigns the RBs in an ascending order whereby its $k_{1}$-th incoming WT is assigned the RB $r_{k_{1}} \in \mathcal{R}$. In contrast, the second RT assigns the RBs in a descending order whereby its $k_{2}$-th incoming WT is assigned the RB $r_{N-k_{2}+1} \in \mathcal{R}$. From this simple assignment scheme it is seen that a hit will occur if and only if the total network load $K=k_{1}+k_{2}>N$.

Unfortunately, in contrast with the case of $M=2$, when the number of RTs $M>2$, it is easy to show that there is no scheme that can eliminate the possibility of hits without compromising the availability of the $N$ RBs to all RTs. In order to address this difficulty, in the next section we will provide a scheme that enables the RTs to assign the RBs in a way that results in a relatively small number of hits. To enhance our scheme, we assume that the RTs are able to detect hit occurrences. Using its knowledge of the assignment sequences of other RTs in the network, each RT identifies the RTs with which assignment conflicts have occurred and uses this information to avoid future hits.

\section{The Resource Block Assignment Scheme}

Consider the situation in which the communication between the BS and the WTs is assisted by $M \leq \phi(P-1)$ RTs, where $P$ is a prime integer. Furthermore, we make the mild assumptions that the number of available $\mathrm{RBs}$ is $P-1$ and that the number of WTs, $K \leq P-1$; cf. Section III. In the proposed scheme, each RT $i \in\{1, \ldots, M\}$ uses a group generator $g_{i}$, which is one of the $\phi(P-1)$ primitive roots of $P$; cf. Section II. If the RTs operate in a decode-andforward (DF) mode, the group generator can be assigned by the BS and embedded in the first BS-RT communication. Alternatively, if the RTs operate in an amplify-and-forward mode, they will not be able to decode the BS signals. In this case, the group generators can be autonomously selected by the RTs from locally built-in look-up tables. The latter strategy can be also used for RTs employing DF relaying. Whether the group generators are provided by the BS or locally selected from look-up tables, providing the RTs with these generators incurs negligible resource consumption and requires no further monitoring from the BS. Similar to the RTs, each BS uses a group generator to generate RBs assignment sequence to the WTs directly connected to it. Upon entering the service domain of the RT, a WT $k_{1}$ is assigned an $\mathrm{RB} g_{i}^{k_{1}}(\bmod P)$. 
The above technique is readily extensible to the case in which the RTs assign multiple RBs to a single WT with high traffic requirements. In particular, such an assignment can be regarded as an assignment of one $\mathrm{RB}$ to multiple incoming WTs. The fact that each primitive root spans the entire set of available RBs implies that the proposed scheme is well suited to situations in which the distribution of WTs is not uniform. To see this, we note that, because this scheme does not constrain the number of RBs used by any RT, it enables RTs to assist as many WTs as the number of unassigned RBs. Hence, this scheme allows a particular RT to assist more than the average WT load, $K / M$.

Using this assignment scheme, when $M$ is strictly less than $\phi(P-1)$, the optimal choice of the primitive roots must ensure that the number of hit occurrences at which one RB is assigned to multiple WTs is minimized. (When $M=\phi(P-1)$ the optimization of primitive roots is trivial; all the primitive roots are assigned to all the RTs.) However, further minimization of the number of hits can be obtained by using the result in Lemma 1. In particular, using this result it can be seen that the number of hits can be minimized, not only over the sequences generated by the primitive roots, but also over cyclically shifted versions thereof. In this case, in addition to a primitive root $g_{i}$, each RT is assigned a cyclic shift $s_{i} \in\{0, \ldots P-2\}$. Using the primitive-root-cyclic-shift (PRCS) pair $\left(g_{i}, s_{i}\right)$ the $i$-th RT assigns the RB $r_{\ell}$ to its $k_{1}$-th incoming WT, where

$$
\ell=g_{i}^{k_{1}+s_{i}}(\bmod P) .
$$

In Section VI we will show that augmenting the search for sequences to include the cyclic shifts $\left\{s_{i}\right\}$ can have a significant impact on the number of hits.

When cyclic shifts are not used in constructing the assignment sequences, the number of hits is minimized over $\left(\begin{array}{c}\phi(P-1) \\ M\end{array}\right)$ primitive roots. However, by introducing the shifts, the number of possible sequences is increased to $(P-1)^{M}\left(\begin{array}{c}\phi(P-1) \\ M\end{array}\right)$. Although relatively large, this number is significantly smaller than $\left(\begin{array}{c}(P-1) ! \\ M\end{array}\right)$, which is the number of distinct assignment sequences. To appreciate this gain in design complexity, we consider the case of $P=41$ and $M=3$. In this case, the number of sequences generated by the proposed method is $3.548 \times 10^{7}$, whereas the total number of distinct sequences is greater than $9 \times 10^{142}$.

Since for a given number of RBs, the assignment sequences have to be designed once for each $M \leq \phi(P-1)$, the proposed method for designing assignment sequences incurs a negligible long term computational cost.

We will show in Section VI that the proposed method yields favourable autonomous RB assignments. However, these assignments are only optimal with respect to the set of cyclically generated sequences, but not necessarily optimal with respect to the set of all possible sequences. In the following section we will introduce a metric which will enable efficient optimization of PRCS pairs.

\section{A. A metric for selecting PRCS pairs}

In order to facilitate the selection of the assignment sequences, it is useful to associate with each sequence a metric that reflects the number of hits that result from using this sequence. We begin by associating a $(P-1) \times(P-1)$ load matrix with each RT $i$, a primitive root $g_{i}$ and a cyclic shift $s_{i}$. We will denote this matrix by $X\left(g_{i}, s_{i}\right), i \in\{1, \ldots, M\}$, and $s_{i}=0, \ldots, P-2$. The row and column indices of this matrix represent the RB and the load level of RT $i$, respectively. In particular, the $\left(\ell_{1}, \ell_{2}\right)$-th entry of $X\left(g_{i}, s_{i}\right)$ represents the binary state of RB $\ell_{1}$ when the load level of RT $i, k_{i}$, is $\ell_{2}$. Setting this entry equal to 1 indicates that $\mathrm{RB} \ell_{1}$ is assigned by RT $i$, when its load, $k_{i}=\ell_{2}$, whereas setting this entry equal to 0 indicates that $\mathrm{RB} \ell_{1}$ is not assigned by $\mathrm{RT} i$, when $k_{i}=\ell_{2}$. Using this notation, the $\left(\ell_{1}, \ell_{2}\right)$-th entry of $X\left(g_{i}, s_{i}\right)$ can be written as shown in (2).

$$
\left[X\left(g_{i}, s_{i}\right)\right]_{\ell_{1}, \ell_{2}}= \begin{cases}1 & \text { if } \quad \ell_{1} \in \mathcal{L}\left(g_{i}, s_{i}, \ell_{2}\right) \quad \text { and } \\ 0 & \text { otherwise }\end{cases}
$$

$\mathcal{L}\left(g_{i}, s_{i}, \ell_{2}\right)=\left\{g_{i}^{1+s_{i}}(\bmod P), \ldots, g_{i}^{\ell_{2}+s_{i}}(\bmod P)\right\}$.

As an example, suppose that the PRCS pairs $\left(g_{i}, s_{i}\right)$ and $\left(g_{j}, s_{j}\right)$ yield the assignment sequences $(1,4,3,2)$ and $(2,3,1,4)$ for the $i$-th and $j$-th RTs, respectively. The corresponding load matrices, $X\left(g_{i}, s_{i}\right)$ and $X\left(g_{j}, s_{j}\right)$, are

$$
X\left(g_{i}, s_{i}\right)=\left[\begin{array}{llll}
1 & 1 & 1 & 1 \\
0 & 0 & 0 & 1 \\
0 & 0 & 1 & 1 \\
0 & 1 & 1 & 1
\end{array}\right], \quad X\left(g_{j}, s_{j}\right)=\left[\begin{array}{cccc}
0 & 0 & 1 & 1 \\
1 & 1 & 1 & 1 \\
0 & 1 & 1 & 1 \\
0 & 0 & 0 & 1
\end{array}\right] .
$$

The inner product of the $k_{1}$-th column of $X\left(g_{i}, s_{i}\right)$ and the $k_{2}$-th column of $X\left(g_{j}, s_{j}\right)$ yields the number of hits when RTs $i$ and $j$ are loaded by $k_{1}$ and $k_{2}$ WTs, respectively. This inner product is given by the $\left(k_{1}, k_{2}\right)$-th entry of what we call the pairwise hit matrix $H_{i, j}$, where

$$
H_{i, j}=X^{T}\left(g_{i}, s_{i}\right) X\left(g_{j}, s_{j}\right) .
$$

For the load matrices given in (3), the resulting pairwise hit matrix between RTs $i$ and $j H_{i, j}$ is:

$$
H_{i, j}=\left[\begin{array}{cccc}
0 & 0 & 1 & 1 \\
0 & 0 & 1 & 2 \\
0 & 1 & 2 & 3 \\
1 & 2 & 3 & 4
\end{array}\right]
$$

For instance, from this matrix, the number of hits when the $i$-th RT is loaded by 2 WTs and the $j$-th RT is loaded by 3 WTs is given by the entry $\left[H_{i, j}\right]_{2,3}=1$.

Let us denote the load of the $i$-th RT by $k_{i}, i=1, \ldots, M$, and denote the total number of hits observed by the $M$ RTs by $Z\left(k_{1}, \ldots, k_{M}\right)$. Then, it can be seen that

$$
Z\left(k_{1}, \ldots, k_{M}\right)=\sum_{i=1}^{M-1} \sum_{j=i+1}^{M}\left[H_{i, j}\right]_{k_{i}, k_{j}} .
$$

This expression is useful to determine the number of hits when the instantaneous load of each RT is known. However, in practical cellular networks the loads of RTs may vary rapidly, which renders timely communication between RTs costly and unrealistic. Furthermore, in the absence of centralized coordination, which is the case considered herein, autonomous identification of the loads of all RTs in the cell is not guaranteed to be successful. Thus, to enable a flexible autonomous RB assignment that adapts to bursty traffic and both uniform and nonuniform WT distributions, the metric for choosing the 
PRCS pairs must not depend on the instantaneous load. Hence, for the proposed scheme we will choose the PRCS pairs that minimize the average, rather than the instantaneous, number of hits. In this case, the metric for selecting the PRCS pairs can be expressed as

$$
C(K)=\frac{1}{\left(\begin{array}{l}
K-1 \\
M-1
\end{array}\right)} \sum_{\left(k_{1}, \ldots, k_{M}\right) \in \mathcal{S}_{K}} Z\left(k_{1}, \ldots, k_{M}\right),
$$

where $\mathcal{S}_{K} \triangleq\left\{\left(k_{1}, \ldots, k_{M}\right) \mid \sum_{i=1}^{M} k_{i}=K, k_{i} \in \mathbb{N}, i=\right.$ $1, \ldots, M\}$. Hence, $C(K)$ is equal to the number of hits averaged over all possible $M$ partitions of $K$. Note that, since each PRCS pair yields a particular RB assignment sequence, $C(K)$ is a deterministic quantity that can be computed a priori independently of the channel realizations. The computation of $C(K)$ can be performed off-line and the optimal PRCS pairs can be stored in a look-up table either at the BS or at the RTs.

\section{B. Computational complexity of $C(K)$}

To assess the computation complexity of evaluating $C(K)$, we note that the entries of the matrices $\left\{X\left(g_{i}, s_{i}\right)\right\}$ take on values from $\{0,1\}$. Hence, the cost of the entry-wise multiplications in (4) is negligible, and only addition operations contribute to the computational cost of evaluating $C(K)$. In particular, the number of addition operations required for computing each of the $M(M-1) / 2$ matrices $H_{i, j}$ in (4) is $(P-1)^{2}(P-2)$ and the number of addition operations required for computing $Z$ in (6) is $M(M-1) / 2-1$. To compute the number of addition operations required to compute $C(K)$ in (7), we note that the cardinality of the set $\mathcal{S}_{K}$ is equal to the number of ways an integer can be partitioned into $M$ ordered parts. This number is given by $\left(\begin{array}{l}K-1 \\ M-1\end{array}\right)$ [13]. Hence, the total number of addition operations required to compute $C(K)$ is bounded by

$$
\frac{1}{2} M(M-1)\left((P-1)^{2}(P-2)+\left(\begin{array}{l}
K-1 \\
M-1
\end{array}\right)\right) .
$$

From (8), we note that computing $C(K)$ involves a reasonable number of additions for small-to-moderate values of $K$ and $M$. However, because of the last term, $C(K)$ becomes computationally prohibitive when $K$ and $M$ are large.

\section{Sequence selection}

When the system load, $K$, is known a priori, the metric $C(K)$ provides an efficient means for assessing the number of hits resulting from choosing a particular set of PRCS pairs for generating the assignment sequences of the RTs. However, when the system load is not known, an alternative is to select one set of PRCS pairs to be used with all possible system loads. Such a set can be chosen to minimize the average of $C(K)$ over all $K \in\{1, \ldots, N\}$, which is proportional to $\sum_{K=1}^{N} C(K)$. In Section VI we will show that the set of PRCS pairs that minimizes $\frac{1}{N} \sum_{K=1}^{N} C(K)$ yields a performance close to that yielded by the set of PRCS pairs that minimize $C(K)$ at each $K$. In Section V, we will propose a technique by which the number of hits can be reduced. This technique relies on incorporating additional information to which the RTs may have access. Even though in this case the metric $\sum_{K=1}^{N} C(K)$ only represents an upper bound on the number of hits, in Section VI we will show that the sequences chosen according to this metric still yield favourable performance.

\section{Further Enhancement of the Resource Block ASSIGNMENT SCHEME}

In the previous section, we provided an efficient scheme for designing RB assignment sequences for RTs in the absence of CQIs. In this scheme, a set of PRCS pairs is selected to ensure that the sequences generated by (1) yield a small number of hits. In this section, we will exploit the deterministic nature of the proposed assignment sequences to further reduce the number of hits. In particular, we will show that, using these sequences, the RTs will be able to identify the sequence with which a hit has occurred, to infer the loads of other RTs, and to subsequently utilize this information to avoid future hits. To do this, we assume that the $i$-th RT is aware of the PRCS pairs $\left\{\left(g_{j}, s_{j}\right)\right\}_{j=1, j \neq i}^{M}$, of the other $M-1$ RTs in addition to its prescribed $\left(g_{i}, s_{i}\right)$-pair. For randomly generated sequences, the RTs do not have information about the RB assignments of other RTs and hence will not be able to avoid future hits using the technique described below.

\section{A. Hit Source Identification and Avoidance (HIA)}

With the set of PRCS pairs $\left\{\left(g_{i}, s_{i}\right)\right\}_{i=1}^{M}$ available, each RT can generate the assignment sequences utilized by other RTs. When an RT detects a hit, it examines the sequences of the other RTs. First, it determines the RBs on which no hits were detected in the past and subsequently eliminates a subset of RTs from the set of potential hit sources. However, this procedure may not be sufficient to determine the exact sequence with which a hit has occurred. To resolve this ambiguity, the RT waits for more hits to occur. Incorporating this information, the RT is able to reduce the number of elements in its ambiguity set. We will refer to this procedure as the hit identification and avoidance (HIA) algorithm, which can be summarized in the following steps:

- An RT identifies a hit on a certain RB, say RB.

- The RT compares the set of its previously assigned RBs with the set of RBs preceding $\hat{R B}$ in the assignment sequences of all other RTs.

- If the intersection of the set of previously assigned RBs and the set of RBs preceding $\hat{\mathrm{RB}}$ in the assignment sequence of a certain RT is not empty, that RT is excluded from the set of potential hit sources. This comparison is repeated for all RTs in the system.

- If the hit source is identified, i.e., there is one element in the set of potential hit sources, the RBs preceding $\hat{R B}$ in the assignment sequence of the hit source are avoided in future assignments.

- If the hit source is not identified, the RT continues to assign the RBs according to its sequence until another hit occurs, and the algorithm is repeated.

From the above description, it can be seen that the operation of the HIA algorithm depends solely on the current loads of the RTs, but not on the instants at which the WTs access the system. Furthermore, it can be verified that the operation of the HIA algorithm is not affected if some RTs fail to identify the hit source. This is because, in identifying the hit source, the HIA algorithm depends on the RBs that are assigned without hits. However, if an RT does not identify a hit source, it assigns RBs that are already assigned by other RTs. Being already assigned, those RBs are discarded by the 


\begin{tabular}{c|cccccccccccccccc}
$\left(g_{1}, s_{1}\right)$ & 3 & 9 & 10 & 13 & 5 & 15 & 11 & 16 & 14 & 8 & 7 & 4 & 12 & 2 & 6 & 1 \\
\hline$\left(g_{2}, s_{2}\right)$ & 5 & 8 & 6 & 13 & 14 & 2 & 10 & 16 & 12 & 9 & 11 & 4 & 3 & 15 & 7 & 1 \\
\hline$\left(g_{3}, s_{3}\right)$ & 6 & 2 & 12 & 4 & 7 & 8 & 14 & 16 & 11 & 15 & 5 & 13 & 10 & 9 & 3 & 1
\end{tabular}

HIA algorithm of other RTs. To better explain the operation of the HIA algorithm, we now provide an example for a system with three RTs.

Example 1: Let $M=3, P=17, s_{i}=0, i=1,2,3$, and $g_{1}=3, g_{2}=5$ and $g_{3}=6$, where $g_{1}, g_{2}$ and $g_{3}$ belong to the set of primitive roots of 17 . The sequences generated by (1) with $\left\{\left(g_{i}, s_{i}\right)\right\}_{i=1}^{3}$ are given in the table above.

Let us consider the case in which the loads of the three RTs are given by $k_{1}=4, k_{2}=3$ and $k_{3}=0$. First, RT 2 assigns RB 6 and no hit is detected. Since this RB is the first to be used by RT 3 , RT 2 realizes that $k_{3}=0$. Now, suppose that $k_{2} \rightarrow k_{2}+1$ and, based on its sequence, RT 2 assigns RB 13 to the incoming WT. Since this RB is already assigned by RT 1, both RTs detect a hit, and RT 2 identifies RT 1 as the hit source. However, because the RBs 3, 9 and 10 are not used yet by RTs 2 and 3, RT 1 is unable to determine the RT with which the hit at RB 13 has occurred. Hence, RT 1 continues to assign the RBs according to its sequence. Suppose that $k_{1} \rightarrow k_{1}+1$ and RT 1 assigns RB 5 to the incoming WT. RT 1 detects a hit, but is still unable to determine the hit source. When $k_{1} \rightarrow k_{1}+1$, RT 1 assigns RB 15 to the incoming WT and no hit is detected. Since RT 3 assigns RB 15 before RB 13 and no hit was detected at RB 15, RT 1 concludes that the hit source was RT 2.

The HIA algorithm can be systematically implemented by any RT $i \in\{1, \ldots, M\}$, using the flow chart in Figure 2. In this flow chart, the set containing the RBs already assigned by RT $i$ is denoted by $\mathcal{R}_{i}$, and the sets of RBs preceding RB (the RB at which a hit occurred) in the assignment sequence of RT $j$ are denoted by $\breve{\mathcal{R}}_{j}, j=1, \ldots, M, j \neq i$. The potential hit sources set is denoted by $\overline{\mathcal{R}}_{i}$. The index of incoming WTs is denoted by $k$ and the hit source index is denoted by $s$.

For initialization, the sets $\mathcal{R}_{i}$ and $\breve{\mathcal{R}}_{j}, j=1, \ldots, M, j \neq i$, are set to be empty. The set $\overline{\mathcal{R}}_{i}$ is set to contain all the other RTs; i.e., $\overline{\mathcal{R}}_{i}=\{j\}_{j=1, j \neq i}^{M}$. The WT index, $k$, and the hit source index, $s$, are set to zero. Upon the arrival of a new WT, the index $k$ is increased to $k+1$ and the new WT is assigned $\mathrm{RB} r=g_{i}^{k+s_{i}}(\bmod P)$. If no hit is observed when $\mathrm{RB} r$ is assigned, $\mathcal{R}_{i} \leftarrow \mathcal{R}_{i} \bigcup\{r\}$. Alternatively, if a hit is observed, the set $\breve{\mathcal{R}}_{j}$ is set to be equal to the set of RBs preceding $\hat{\mathrm{RB}}$ in the assignment sequence of RT $j, j=1, \ldots, M, j \neq i$. At this point, two possibilities can be considered: 1) the hit source, RT $s$, is immediately identified; and 2) the hit source is inferred from the RBs assignment sequences.

In the first case, RT $i$ distinguishes RT $s$ using, for instance, its modulation scheme. Using this information, RT $i$ avoids assigning the RBs preceding $\hat{R B}$ in the assignment sequence of RT $s, \breve{\mathcal{R}}_{s}$. As will be shown in Section VI, hit avoidance in this case is more effective than in the case considered next, wherein the hit source is not immediately identified, but is inferred from the assignment sequences.

In the second case, the hit source, RT $s$, is not immediately identified. To infer the hit source from the assignment sequences, RT $i$ compares $\mathcal{R}_{i}$ with the sets $\left\{\breve{\mathcal{R}}_{j}\right\}_{j=1, j \neq i}^{M}$. For

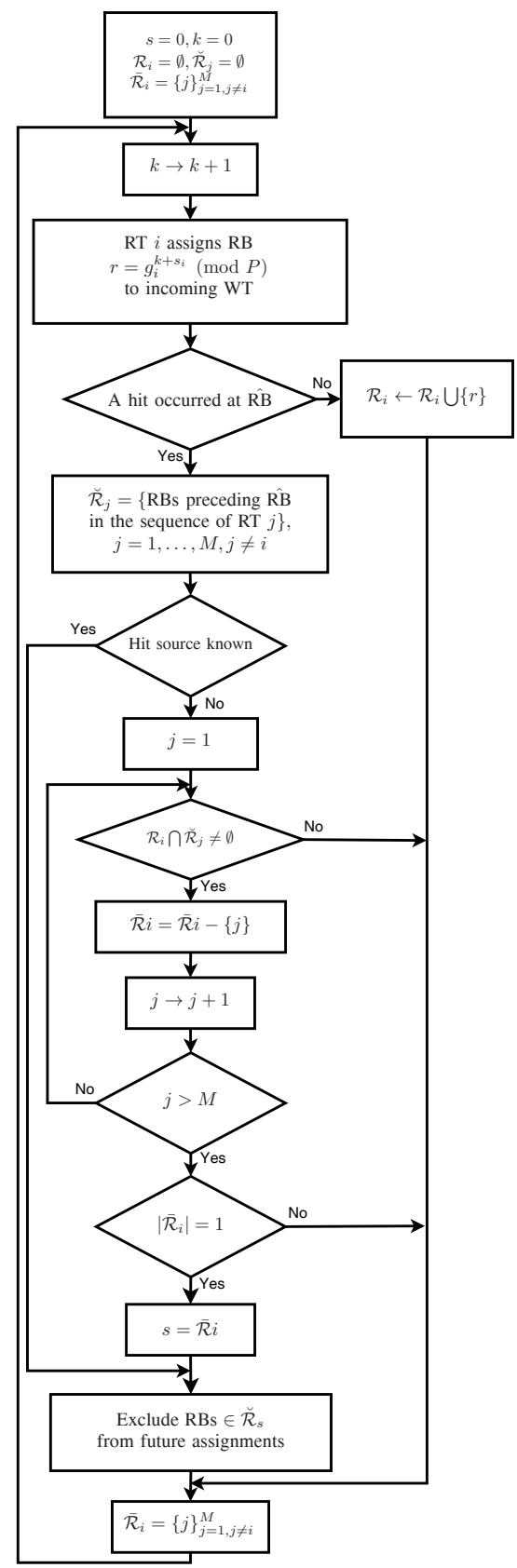

Fig. 2. Flow chart used by RT $i$ for hit identification and avoidance (HIA).

each $j \neq i$, if $\mathcal{R}_{i} \bigcap \breve{\mathcal{R}}_{j} \neq \emptyset, \overline{\mathcal{R}}_{i} \leftarrow \overline{\mathcal{R}}_{i}-\{j\}$. If $\left|\overline{\mathcal{R}}_{i}\right|=1$, RT $i$ declares the remaining element in $\overline{\mathcal{R}}_{i}$ to be the hit source, RT $s$. Similar to the case in which the hit source is immediately identified, after determining $s$, RT $i$ avoids assigning the RBs preceding $\hat{R B}$ in the assignment sequence of RT $s$. If the HIA is unable to identify the hit source, i.e., $\left|\overline{\mathcal{R}}_{i}\right|>1$, RT $i$ continues to assign the RBs according to its sequence until another hit occurs.

Note that, if the HIA algorithm of a particular RT does not identify the hit source, this RT will not be able to avoid future hits and will hence assign the RBs using its 
assignment sequence, as it would, had the HIA algorithm been not employed. In both cases, the RT that was first to assign $\hat{\mathrm{RB}}$ retains it. The other RT, which was second to attempt assigning $\hat{\mathrm{RB}}$, relinquishes it and assigns the following $\mathrm{RB}$ in its assignment sequence.

\section{B. Computational complexity of the HIA algorithm}

First we note that the HIA algorithm does not involve any multiplications, and its computational complexity arises only from the comparison of the assignment sequences when a hit is detected. Each sequence comparison requires at most $P-1$ subtractions. Since the number of RTs is less than or equal to $\phi(P-1)$, for each hit occurrence, the overall complexity of the HIA algorithm is bounded by $(P-1) \phi(P-1) \ll$ $(P-1)^{2}$ subtractions. Hence, the computational complexity of the HIA algorithm is upper bounded by $(P-1)^{2}$ subtraction operations, which can be easily accommodated by standard wireless devices.

\section{Simulation Results}

In this section, the performance of the proposed assignment sequences with and without the HIA scheme is compared with the performance of random [3], PN-based, and the optimal centralized schemes at different relative loads $\frac{K}{P-1}$; i.e., the ratio of the currently assigned RBs to the total number of RBs in the system.

Random assignment of RBs to WTs has been used in various distributed systems such as wireless sensor networks, wherein the nodes selfishly contend for the available RBs. In contrast, for frequency hopping systems, the assignment of RBs in the hopping patterns has been traditionally based on the decimal representation of the states of PN sequence generators. Both random and PN-based assignments can be utilized to generate assignment sequences for the relay-assisted OFDMAbased systems considered herein.

For PN-based assignments, the set of states of PN sequence generators possess a cyclic group structure that facilitates their generation. In particular, for a given integer, $n>1$, there are $\frac{\phi\left(2^{n}-1\right)}{n}$ primitive polynomials that generate length$\left(2^{n}-1\right)$ periodic PN sequences [14]. Notice that the length of the sequence generated by this technique is odd, whereas that generated by the proposed scheme is even. Hence, for comparison, we will consider prime integers of the form $P=2^{n}-1$, which yield sequence lengths that differ by one. In addition to the PN and random based assignment schemes in which CQIs are assumed not to be available, we compare our results with the optimal centralized scheme in which the BS has perfect CQI information and performs an exhaustive search over all possible RB assignments.

For ease of exposition, we restrict our attention to the case in which the number of RTs is $M=3$. For each system load, $K$, the performance is measured by the average number of hits over possible load combinations; i.e., the average overall ordered triples $\left(k_{1}, k_{2}, k_{3}\right) \in\left\{\left(k_{1}, k_{2}, k_{3}\right) \mid \sum_{i=1}^{3} k_{i}=\right.$ $\left.K, k_{i} \geq 0\right\}$.

Example 2: In this example we consider the cases where $P=17$ and $P=31$. The HIA technique of Section $\mathrm{V}$ is not used and for each case, the assignment sequences are generated by (1) with the PRCS pairs that minimize

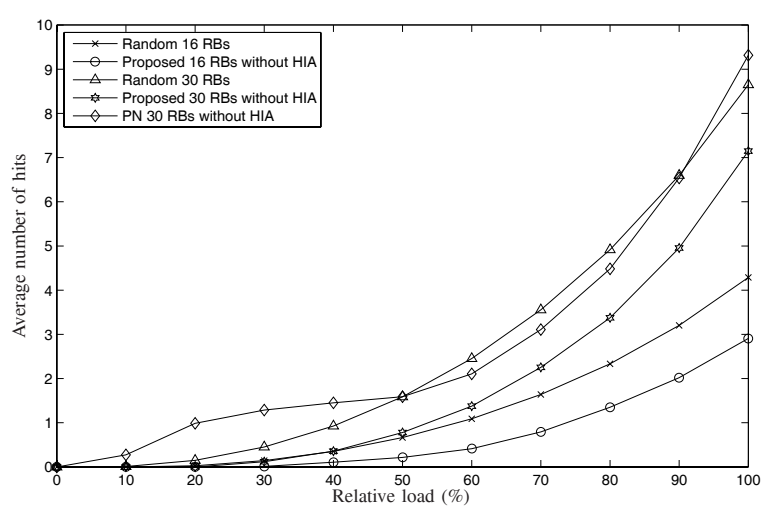

Fig. 3. Comparison between proposed assignment sequences, the random assignment scheme proposed in [3] and the PN based assignment sequences for $N=P-1$ RBs with $P=17$ and $P=31$.

$\sum_{K=1}^{P-1} C(K)$; cf. (7). In all curves, the cyclic shifts were set to 0 . For $P=17$, the optimal roots were found to be: $g_{1}=3$, $g_{2}=11, g_{3}=14$, whereas for $P=31$ the optimal roots were found to be: $g_{1}=3, g_{2}=11, g_{3}=21$. Although it may be possible to optimize the choice of the primitive roots and the cyclic shifts for each $K$, our investigations indicate that this approach yields marginal improvement in performance and does not warrant the additional computational complexity. This observation will be elaborated on in Example 7 below.

For the case of $P=31$, Figure 3 shows a comparison between the performance of the proposed, random [3] and the PN based schemes, and for the case of $P=17$, Figure 3 shows a comparison between the performance of the proposed and random schemes only. This is because, when $N=P-1=16$, the length of the closest PN sequences is $2^{4}-1=15$. These sequences are generated by $\frac{\phi(15)}{4}=2$ primitive polynomials, which are not enough to support the 3 RTs.

From this figure, it can be seen that the proposed sequences yield a significant reduction in the average number of hits. For instance, when $\frac{K}{P-1}=90 \%$, the average number of hits produced by the proposed scheme is 2 and 4.9 for $P=17$ and $P=31$, respectively. The corresponding numbers of hits produced by the technique in [3] are 3.2 and 6.6 , and when $P=31$, the PN-based assignment produced an average number of hits of 6.5 .

Example 3: In this example, we provide additional comparisons between the proposed and the PN-based assignment schemes with and without using the HIA technique of Section V. For the proposed sequences, $P=127$ yielding $N=126$ and for the PN-based scheme, $n=7$ yielding $N=127$. The primitive polynomials of the PN sequences were selected to be those that minimize the number of hits. For simplicity, for the PN-based sequences, the initial state is chosen to be one, and for the proposed sequences the shifts are set to be zero. The RTs are assumed to know the assignment sequences of other RTs and the hit sources are assumed to be immediately identified. From Figure 4, it can be seen that the proposed assignment sequences yield a smaller average number of hits than their PN-based counterparts. For instance, when $\frac{K}{P-1}=90 \%$ the proposed scheme with HIA yields an average of 4.6 hits, whereas the PN-based scheme with HIA yields an average of 10.1 hits. The performance advantage of 


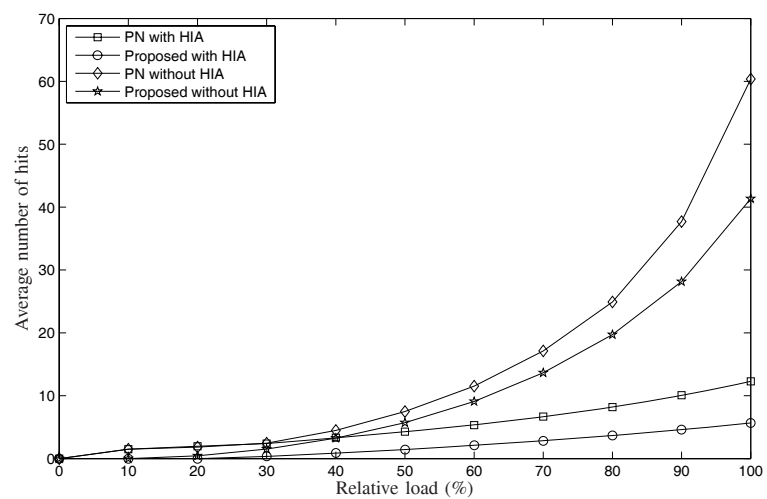

Fig. 4. Comparison between the PN-based and the proposed assignment schemes for $P=127$ and $n=7$ when the cyclic shifts are set to zero with and without immediate HIA.

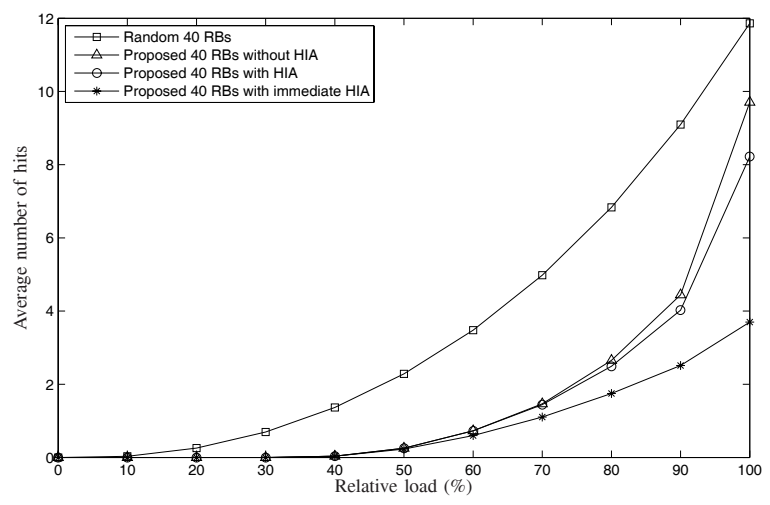

Fig. 5. Comparison between random assignment scheme proposed in [3] and the proposed assignment for $N=40$ RBs (i.e., $P=41$ ) with and without HIA and with immediate HIA.

the proposed scheme can be attributed to the fact that this scheme spans a set of sequences larger than that spanned by the PN generation technique.

Finally, it is to be noted that, because primes are denser than numbers of the form $2^{n}$ in the set of integers [11], the proposed scheme covers a selection of $N=|\mathcal{R}|$ larger than that covered by the PN-based assignment scheme; cf. Section III.

Example 4: In this example we consider a setup similar to the one in the previous example, but with $P=41$. However, we consider two scenarios for the HIA technique, one in which the hit sources are immediately identified and another in which the hit sources are identified with the technique of Section V. Notice that the HIA technique of Section V cannot be applied to the assignment sequences proposed in [3] because of their random structure.

Figure 5 demonstrates the impact of using the HIA technique of Section V. For comparison, the average number of hits generated by the random technique is also shown in this figure. When $\frac{K}{P-1}=90 \%$, the average number of hits produced when no hit avoidance is used is 4.44 . Using hit avoidance, this number is reduced to 4.03 when the hit source is identified with the HIA technique of Section V and to 2.5 when the hit source is identified immediately. The average number of hits when the technique in [3] is used is 9.1.

The metric in (7) offers a convenient means for optimizing the primitive roots and the cyclic shifts of the assignment

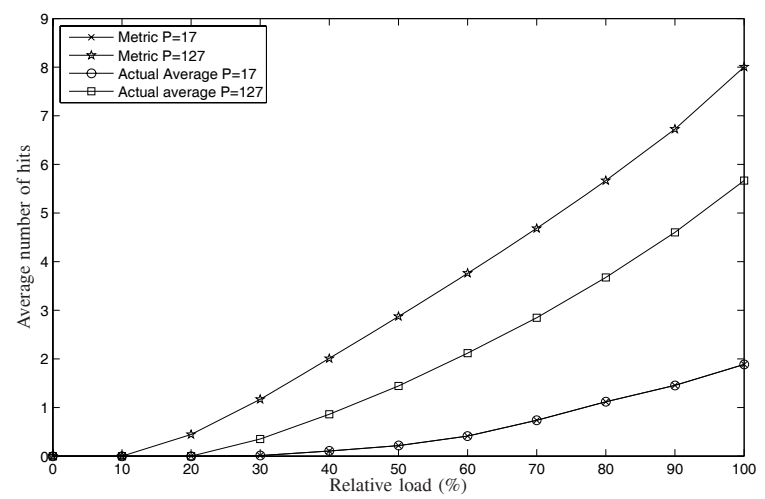

Fig. 6. Comparison between metric minimizing roots and actual average minimizing roots for $P=17$ and $P=127$ with immediate HIA.

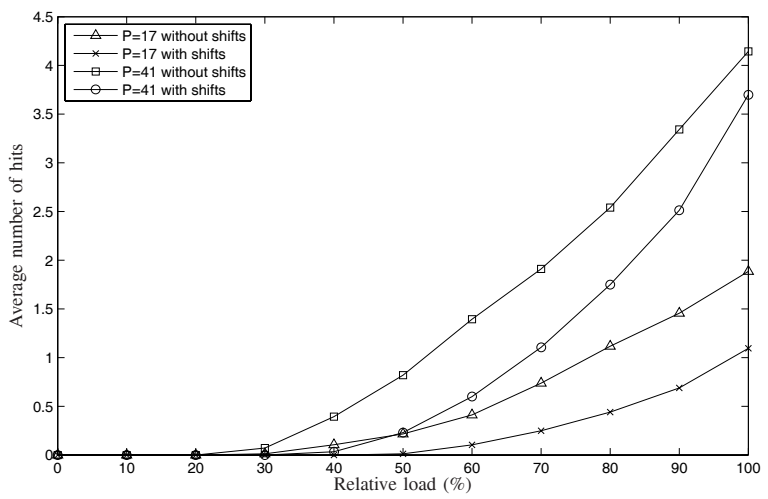

Fig. 7. Proposed assignment scheme for $P=17$ and $P=41$ with and without cyclic shifts with immediate HIA.

sequences. However, this metric does not take the hit source identification and avoidance into consideration. Hence, when the HIA technique is used, the proposed metric yields an upper bound on the actual number of hits. In the following example, we will compare the performance of the HIA scheme when the primitive roots are chosen in two ways: to minimize the metric and to minimize the actual average number of hits.

Example 5: To illustrate the usefulness of the metric, in Figure 6 we compare the performance of the HIA scheme when $P=17$ and $P=127$. The cyclic shifts are set equal to zero; i.e., $s_{1}=s_{2}=s_{3}=0$.

From this figure, it can be seen that the primitive roots selected with the metric yield close-to-optimal performance when $P$ is small. However, as $P$ increases, the performance of these roots deviates from that of the optimal ones.

Example 6: In this example, we show the performance gain resulting from incorporating cyclic shifts in the design of the assignment sequences. We consider the case in which $P=$ 17 and $P=41$ and the HIA technique is used assuming immediate hit source identification.

From Figure 7, it can be seen that, in comparison with the case in which the cyclic shifts are fixed, the joint optimization of shifts and primitive roots yields a significant reduction in the average number of hits. For instance, when $\frac{K}{P-1}=90 \%$, using cyclically shifted assignment sequences with HIA results in an average of 0.69 and 2.5 hits for $P=17$ and $P=41$, respectively, whereas when no shifts are employed, the corre- 


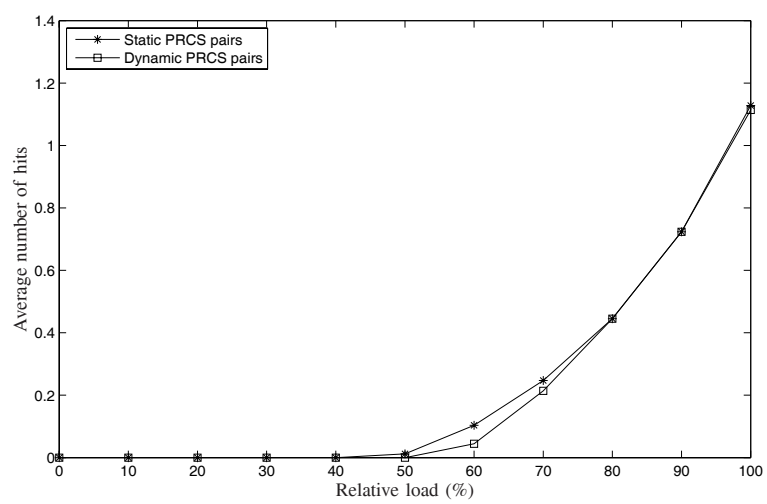

Fig. 8. Comparison between the performance of the PRCS pairs that minimize $\frac{1}{N} \sum_{K=1}^{N} C(K)$ and that of the PRCS pairs that minimize $C(K)$ for each system load $K$. The number of RBs $N=16$.

sponding number of hits is 1.46 and 3.34, respectively. Hence, the additional degrees of freedom offered by incorporating cyclic shifts in the design yield valuable performance benefits. However, to use the HIA technique in this case, each RT must know the primitive roots and cyclic shifts of other RTs.

Example 7: In this example, we investigate the performance of the proposed RB assignment sequences for a system with 3 RTs and $N=16$ RBs. We compare the case in which the PRCS pairs are chosen to minimize $\frac{1}{N} \sum_{K=1}^{N} C(K)$ with the case in which the PRCS pairs are chosen to minimize $C(K)$ for each system load, $K$. In both cases, the HIA technique is employed assuming immediate hit source identification.

From Figure 8, it can be seen that minimizing $C(K)$ at each $K$ yields marginal reduction in the average number of hits. For instance, for this example, the maximum average reduction is less than 0.06 hits. Hence, it can be seen that minimizing $C(K)$ at each $K$ results in an average performance that is close to that obtained by minimizing $\frac{1}{N} \sum_{K=1}^{N} C(K)$, and does not warrant the additional complexity required for its implementation.

Example 8: In this example, we compare the average signal-to-noise-plus-interference ratio (SINR) achieved by the RB assignment sequences generated by (1), with the average SINR achieved by the random assignments proposed in [3], the PN-based assignments and an upper bound achieved by the optimal centralized scheme. The channel gains between the RTs and the WTs are assumed to be frequency-flat Rayleigh fading with complex Gaussian distribution of unit variance per real dimension as in [15], [16]. In this example, the cyclic shifts are set to zero, the HIA algorithm is not employed and the WTs are assumed to have no hit identification capabilities.

We consider a system with 3 RTs and $N=30$ RBs. The loads of the first and the second RTs are $k_{1}=2$ and $k_{2}=1$. The load of third RT $k_{3}$ is increased from 1 to 25 WTs. For the optimal centralized scheme, the load of the third RT $k_{3}$ is increased only from 1 to 4 WTs; assessing the performance of the optimal centralized scheme for systems with more WTs is computationally Prohibitive. The transmission power of all RTs is set to be $10 \mathrm{~dB}$ and the noise variance is assumed to be unity. To obtain a consistent characterization of the hit, and subsequently interference, avoidance capabilities of the assignment sequences, the SINR must be averaged over

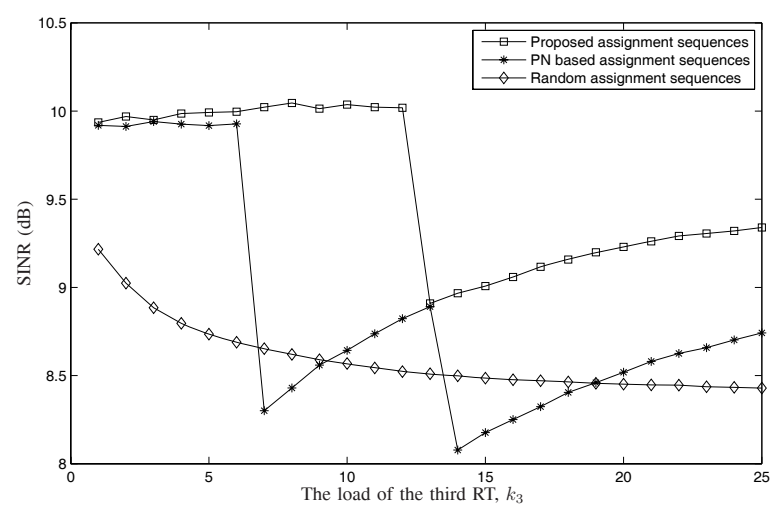

Fig. 9. Comparison between the SINR achieved by the proposed RB assignment sequences, the random assignment sequences [3] and the PN based assignment sequences for $N=30$ and $M=3$.

multiple channel realizations. This is because, although a specific set of assignment sequences might exhibit favourable characteristics for some channel realizations, it may exhibit poor characteristics for other realizations. Hence, in our simulation results the SINR is averaged over 5000 channel realizations.

The performance results of this example are shown in Figure 9. For the centralized scheme, the SINR was found to decrease (almost) linearly from 30 to $22 \mathrm{~dB}$ when the load of the third RT $k_{3}$ increases from 1 to 4 . However, because of the scale of Figure 9 this performance is not shown. From this figure, it can be seen that the proposed RB assignment sequences can provide an average SINR performance improvement in excess of $20 \%$ over random assignments. For the random assignments, the average SINR decreases steadily with the load increase. However, for the proposed and PN based assignment sequences, three regions can be identified. In particular, when the number of WTs is small, $k_{3} \leq 12$ and $k_{3} \leq 6$ for the proposed and PN based assignment sequences, respectively, no hits occur yielding a constant average SINR. When $k_{3}=13$ for the proposed sequences and $k_{3}=7$ and 14 for the PN based assignment sequences, hits occur and the average SINR decreases. As $k_{3}$ increases further, no more hits are observed and the average SINR increases steadily until another hit occurs. It is worth noting from this figure that the performance of the PN based sequences can sometimes fall below the performance of the random assignment sequences.

Example 9: In this example, we compare the average data rate achieved by the proposed sequences with the upper bound achieved by the optimal centralized scheme. The system considered in this example is similar to that considered in the previous one. In particular, the transmission power of all RTs is set to be $10 \mathrm{~dB}$ and the noise variance is assumed to be unity. The loads of the first and second RTs are $k_{1}=1$ and $k_{2}=2$, respectively. The load of the third RT, $k_{3}$, is increased from 1 to 4 WTs. For comparison, we also show the average achievable data rate obtained by the random assignments proposed in [3].

As shown in Figure 10, the centralized scheme provides a performance superior to that of the proposed scheme and the random one. However, the implementation of the centralized 


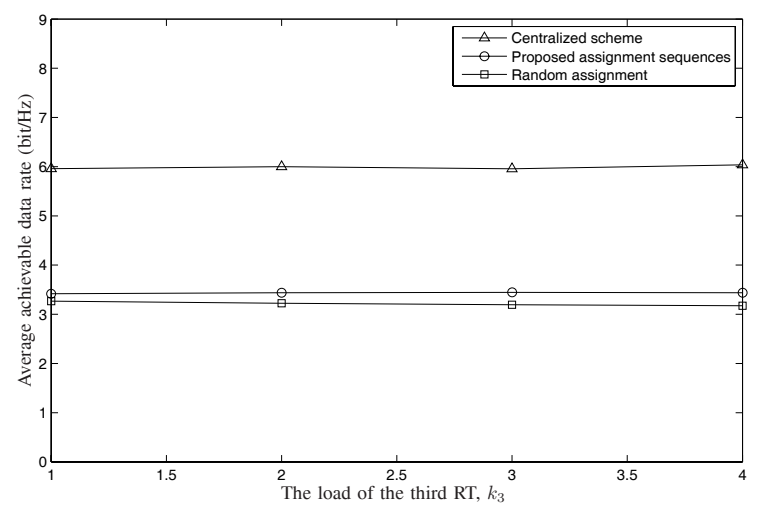

Fig. 10. Comparison between the achievable data rate when the proposed RB assignment sequences, the random assignment sequences [3] and the optimal centralized assignment scheme with perfect CQI knowledge are employed for $N=16$ and $M=3$.

scheme requires the $\mathrm{BS}$ to acquire perfect $\mathrm{CQI}$ information about all RT-WT links and to perform a computationally prohibitive search for each channel realization.

The quality-of-service (QoS) that can be delivered by a system depends on the average SINR observed by the WTs. In particular, a minimum average SINR threshold must be exceeded to ensure the achievability of a target QoS. Since the average SINR tends to decrease when more WTs access the system, the average SINR threshold provides a tradeoff between the QoS and the number of WTs that can be accommodated by the available RBs.

Example 10: In this example, the relative load, $K / N$, that can be accommodated in a three RT system for different average SINR thresholds is compared when the proposed and random assignment sequences are used. Similar to the previous example, the RT-WT channel gains are assumed to be frequency-flat Rayleigh fading with complex Gaussian distribution of unit variance per real dimension and the number of RBs, $N=16$. The transmission power of all RTs is set to be $10 \mathrm{~dB}$ and the noise variance is set to be unity. The threshold SINR is averaged over 5000 channel realizations. To investigate the potential of RB reuse, in this example, $K / N$ is allowed to exceed $100 \%$. The loads of the first and the second RTs are $k_{1}=2$ and $k_{2}=3$, respectively, and the load of the third RT $k_{3}$ is increased from 1 to 16.

From Figure 11, it can be seen that, in comparison with random assignments, the proposed sequences result in a significant increase in the relative load that can be accommodated by the network, without compromising the QoS. For instance, at an average threshold SINR of 9.03, the system with random sequences accommodates $K / N=37.5 \%$, whereas that with the proposed sequences accommodates $K / N=87.5 \%$; a gain of $233 \%$ in the system user capacity. This gain is attributed to the lower interference levels yielded by the proposed cyclically generated sequences.

\section{CONCLUSION}

In this paper a novel technique for autonomous RB assignment in relay-assisted OFDMA-based wireless systems is proposed. This technique eliminates the need for coordination between RTs, and hence is particularly suited for emerging

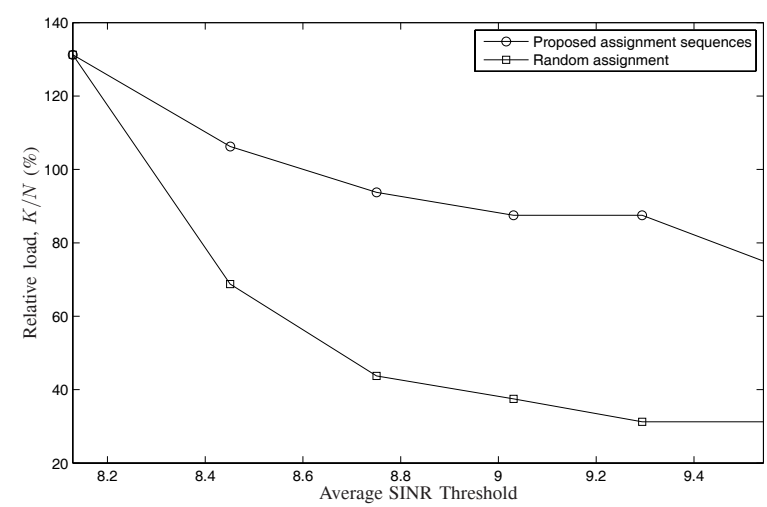

Fig. 11. Comparison between the relative load of the random and proposed assignment sequences at different average threshold SINR levels for $N=16$ RBs.

terminals relaying schemes in which the CQIs are not available. The proposed technique uses cyclic group generation techniques and is shown to offer significant performance improvements over random and PN-based assignments.

\section{APPENDIX A \\ PROOF OF LEMMA 1}

We will prove this lemma by contradiction.

Let $g_{1}$ and $g_{2}$ be two distinct generators of the group $G$. Suppose that the sequence generated by $g_{1}$ is a cyclically shifted version of the sequence generated by $g_{2}$. In this case we can write

$$
g_{1}^{k} \equiv g_{2}^{k+r} \quad(\bmod P),
$$

where $\equiv$ denotes congruence, $k \in\{1, \ldots, P-1\}$ and $r \in$ $\{1, \ldots, P-2\}$ is the cyclic shift. By squaring both sides of (9) we have

$$
g_{1}^{2 k} \equiv g_{2}^{2 k+2 r} \quad(\bmod P) .
$$

Consider the sequence generated by $g_{1}$. The entry of this sequence that lies after $k$ slots from $g_{1}^{k}(\bmod P)$ is $g_{1}^{2 k}$ $(\bmod P)$. Using the assumption that this sequence is a cyclically shifted version of that generated by $g_{2}$, we have from (9) that

$$
g_{1}^{2 k} \equiv g_{2}^{2 k+r} \quad(\bmod P) .
$$

Equating the right hand sides of (10) and (11) yields

$$
g_{2}^{2 k+2 r} \equiv g_{2}^{2 k+r} \quad(\bmod P) .
$$

Multiplying both sides of (12) by $g_{2}^{p-1-2 k-2 r}$, it follows from Fermat's Little Theorem [11] that

$$
1 \equiv g_{2}^{p-1-r} \equiv g_{2}^{-r} \quad(\bmod P) .
$$

The last equation can be satisfied if and only if $r=0$ $(\bmod (P-1))$. Using this in (9) and simplifying yields $g_{1}=g_{2}$, which contradicts the assumption that $g_{1}$ and $g_{2}$ are distinct, which completes the proof of the lemma.

\section{ACKNOWLEDGMENT}

The authors would like to thank Chandra (Sekhar) Bontu, Claude Royer and Jim Womack of Research In Motion, for their valuable comments. The authors would also like to thank the anonymous reviewers for the careful reading and the constructive comments that they provided on our manuscript. 


\section{REFERENCES}

[1] V. Sreng, H. Yanikomeroglu, and D. Falconer, "Coverage enhancement through two-hop relaying in cellular radio systems," in 2002 IEEE Wireless Commun. and Networking Conf.

[2] R. Pabst, B. H. Walke, D. C. Schultz, P. Herhold, H. Yanikomeroglu, S. Mukherjee, H. Viswanathan, M. Lott, W. Zirwas, M. Dohler, H. Aghvami, D. D. Falconer, and G. P. Fettweis, "Relay-based deployment concepts for wireless and mobile broadband radio," IEEE Commun. Mag., vol. 42, pp. 80-89, Sep. 2004.

[3] C. Koutsimanis and G. Fodor, "A dynamic resource allocation scheme for guaranteed bit rate services in OFDMA networks," in Proc. 2008 IEEE Int. Conf. Commun.

[4] A. L. Stolyar and H. Viswanathan, "Self-organizing dynamic fractional frequency reuse in OFDMA systems," in Proc. 2008 IEEE Int. Conf. Comp. Commun.

[5] Z. Kostic and N. Sollenberger, "Performance and implementation of dynamic frequency hopping in limited-bandwidth cellular systems," IEEE Trans. Wireless Commun., vol. 1, pp. 28-36, Jan. 2002.

[6] P. Popovski, H. Yomo, S. Aprili, and R. Prasad, "Frequency rolling: a cooperative frequency hopping for mutually interfering WPANS," in Proc. 2004 ACM Int. Symp. Mobile Ad Hoc Ntwk. Computing.

[7] Z. Kostić, I. Marić, and X. Wang, "Fundamentals of dynamic frequency hopping in cellular systems," IEEE J. Sel. Areas Commun., vol. 19, pp. 2254-2266, Nov. 2001.

[8] O. Mubarek, H. Yanikomeroglu, and S. Periyalwar, "Dynamic frequency hopping in cellular fixed relay networks," in Proc. 2005 IEEE Vehic. Tech. Conf., vol. 5.

[9] J. A. Gallian, Contemporary Abstract Algebra. Houghton Mifflin, 1998.

[10] T. W. Hungerford, Abstract Algebra: An Introduction. Saunders College Publishing, 1997.

[11] W. W. Adams and L. J. Goldstein, Introduction to Number Theory. Prentice-Hall, Inc., 1976.

[12] S. M. Ross, Stochastic Processes. John Wiley, 1996.

[13] G. E. Andrews, The Theory of Partitions. Cambridge University Press, 1976.

[14] R. Lidl and H. Niederreiter, Introduction to Finite Fields and Their Applications. Cambridge University Press, 1994.

[15] T. Y. Al-Naffouri, M. Sharif, and B. Hassibi, "How much does transmit correlation affect the sum-rate scaling of MIMO Gaussian broadcast channels?" IEEE Trans. Commun., vol. 57, pp. 562-572, Feb. 2009.

[16] F. Rusek, A. Lozano, and N. Jindal, "Mutual information of IID complex Gaussian signals on block Rayleigh-faded channels," in Proc. 2010 IEEE Int. Symp. Inf. Theory.

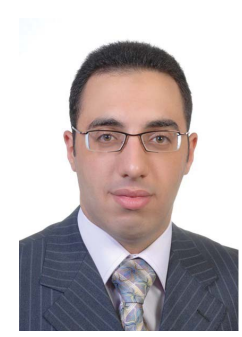

Yaser Fouad received his B.Sc. degree in Electronics and Communications Engineering from the Arab Academy for Science and Technology and Maritime Transport, Cairo, Egypt, in 2009. During his studies, he was ranked first across all departments. He then received a M.A.Sc. degree in Electrical and Computer Engineering from Carleton University, Ottawa, Canada, in 2011, where he is currently working toward the Ph.D. degree in Electrical and Computer Engineering. He is currently a research assistant at Carleton University and a member of the CarletonRIM (Research In Motion) research project.

His research interests include efficient resource allocation in relay-assisted cellular networks, applications of graph theory in the design of graphical medium access control schemes for multihop networks, and power allocation techniques in multiple antenna and multiuser systems.

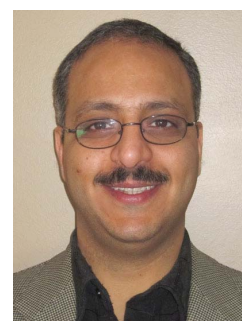

Ramy Gohary (S'02-M'06) received the B.Eng. (Hons.) degree from Assiut University, Egypt in 1996, the M.Sc. degree from Cairo University, Egypt, in 2000, and the Ph.D. degree from McMaster University, Ontario, Canada in 2006, all in electronics and communications engineering. $\mathrm{He}$ received the Natural Sciences and Engineering Research Council visiting fellowship award in 2007.

Dr. Gohary was a visiting fellow with the Terrestrial Wireless Systems Branch, Communications Research Centre, Canada. He is currently the project manager of the Carleton-RIM (Research In Motion) research project.

His research interests include analysis and design of MIMO wireless communication systems, applications of optimization and geometry in signal processing and communications, information theoretic aspects of multiuser communication systems, and applications of iterative detection and decoding techniques in multiple antenna and multiuser systems.

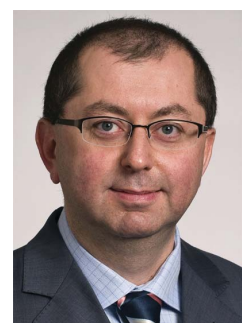

Halim Yanikomeroglu received a B.Sc. degree in Electrical and Electronics Engineering from the Middle East Technical University, Ankara, Turkey, in 1990, and a M.A.Sc. degree in Electrical Engineering (now ECE) and a Ph.D. degree in Electrical and Computer Engineering from the University of Toronto, Canada, in 1992 and 1998, respectively. He was with the R\&D Group of Marconi Kominikasyon Ankara, Turkey, from January 1993 to July 1994.

Since 1998 Dr. Yanikomeroglu has been with the Department of Systems and Computer Engineering at Carleton University, Ottawa, where he is now a Full Professor. Dr. Yanikomeroglu's research interests cover many aspects of the physical, medium access, and networking layers of wireless communications systems and networks. In recent years, Dr. Yanikomeroglu's research has been funded by Huawei (Canada and China), Research In Motion (Canada), Samsung Advanced Institute of Technology (SAIT, Korea), Telus, Communications Research Centre Canada (CRC), Nortel, Ontario Ministry of Research and Innovation, NSERC (Natural Sciences and Engineering Research Council of Canada), and Carleton University.

Dr. Yanikomeroglu is a Distinguished Lecturer for the IEEE Vehicular Technology Society. He has given several plenary and invited talks and over 20 tutorials in the leading international conferences. Dr. Yanikomeroglu is a recipient of the Carleton University Faculty Graduate Mentoring Award in 2010, the Carleton University Graduate Students Association Excellence Award in Graduate Teaching in 2010, and the Carleton University Research Achievement Award in 2009.

Dr. Yanikomeroglu is a member of the Steering Committee of the IEEE Wireless Communications and Networking Conference (WCNC), and has been involved in the organization of this conference over the years, including serving as the Technical Program Co-Chair of WCNC 2004 and the Technical Program Chair of WCNC 2008. Dr. Yanikomeroglu was the General CoChair of the IEEE Vehicular Technology Conference Fall 2010 held in Ottawa (VTC2010-Fall).

Dr. Yanikomeroglu was an editor for IEEE TRANSACTIONS ON WIRELESS COMmunications and IEEE COMmunicATIONS SuRveys \& TUTORIALS, and a guest editor for Wiley Journal on Wireless Communications \& Mobile Computing. He was the Chair of the IEEE Technical Committee on Personal Communications (now called Wireless Technical Committee).

Dr. Yanikomeroglu is spending the 2011-2012 academic year in TOBB University of Economics and Technology, Ankara, Turkey, as a Visiting Professor. Dr. Yanikomeroglu is also an Adjunct Professor at King Saud University's Advanced Technology Research Institute Riyadh, Saudi Arabia. $\mathrm{He}$ is a registered Professional Engineer in the province of Ontario, Canada. 